\title{
Uma Revisão Sistemática da Literatura sobre o uso de Teorias de Aprendizagem em Softwares Educacionais
}

\author{
Mychelline Souto Henrique - UFPE, msh@cin.ufpe.br \\ Carla Taciana Lima Lourenço Silva - UFPE, ctlls@cin.ufpe.br \\ Danielle Rousy Dias da Silva - UFPB, danielle.rousy@gmail.com \\ Patricia Cabral de Azevedo Restelli Tedesco - UFPE, pcart@cin.ufpe.br
}

\begin{abstract}
Resumo: Os Softwares Educacionais (SEs) necessitam de um conjunto de funcionalidades para atender aos aspectos pedagógicos. Atualmente parece não existir um processo de desenvolvimento para SE que se preocupe em elicitar essas funcionalidades e a sua identificação pode ficar comprometida. As teorias de aprendizagem explicam como ocorre o processo de aprendizagem e, portanto, devem ser usadas explicitamente no desenvolvimento dos SEs. No entanto, a comunidade de Informática na Educação carece de evidências sobre o uso de teorias de aprendizagem em SE. Portanto, o principal objetivo deste trabalho é investigar quais as teorias de aprendizagem consideradas no desenvolvimento dos SEs. Para alcançar o objetivo, foi realizada uma Revisão Sistemática da Literatura (RSL), que seguiu um protocolo pré-definido para identificar os estudos primários relevantes. Como resultado, apenas 27,9\% (48 artigos) da amostra analisada (172 artigos) utilizaram teorias de aprendizagem. A teoria de Piaget (construtivismo) é a mais referenciada (20 citações) pelos trabalhos analisados.
\end{abstract}

Palavras-chave: softwares educacionais, teorias da aprendizagem, requisitos, diversas formas de avaliar software educacional, revisão sistemática da literatura

\section{A Systematic Literature Review on the use of Theories of Learning in Education Softwares}

\begin{abstract}
Educational Software (ES) needs a set of functionalities to address pedagogic aspects. Nowadays it seems there isn't a development process tailored for ES and the identification of those functionalities can be jeopardized. Learning theories explain how the learning process happens. However, informatics in education community lacks evidences on the use of learning theories in SE. Therefore, the main objective of this work is to investigate which are the learning theories used in SE. To reach the objective, a Systematic Literature Revision was performed following a pre-defined protocol to identify the relevant primary studies. As result, only $27,9 \%$ (48 papers) of the analyzed sample (172 papers) used learning theories. Piaget's theory (constructivism) is the most referenced one (20 citations) in the analyzed studies.
\end{abstract}

Keywords: educational software, learning theories, requirements, different ways of evaluating educational software, systematic literature revision

\section{Introdução}

A educação tem passado por várias mudanças de paradigmas e as aulas expositivas já não produzem o mesmo efeito no aprendizado (An et al, 2013). Portanto, é necessário repensar as metodologias de ensino. A inclusão da tecnologia nesse processo tem trazido grandes benefícios. Um dos diferenciais dos softwares educacionais, em relação ao software comum, é o fato dele lidar com as necessidades do usuário relacionadas a aprendizagem (Gomes e Padovani, 2005).

Tchounikine (2011) diz que o SE deve ser projetado para promover a aprendizagem considerando objetivos pedagógicos. O trabalho de dissertação de Lima (2012) realizou um mapeamento sistemático sobre o desenvolvimento de SEs, ele afirma que existe uma ausência de metodologia para desenvolver esse tipo de software; 
um dos temas em aberto apontados no trabalho é em relação aos requisitos pedagógicos. Nota-se, porém, que a identificação dos requisitos necessários para este domínio pode ficar comprometida, o que se reflete na qualidade do produto final.

Identificar as funcionalidades relacionadas aos aspectos pedagógicos é essencial no processo de desenvolvimento do SE. Segundo Lima (2012), “[...] as teorias de aprendizagem refletem visões profundamente diferentes sobre como ocorre a aprendizagem e essas visões têm impacto nos softwares educacionais". Diante deste cenário, surgem algumas perguntas, como: quais são os requisitos pedagógicos utilizados nos SEs? Os SEs são fundamentados em teorias ou métodos de aprendizagem? Como é feita a avaliação dos SEs? O trabalho de Ferreira et al. (2014) apresenta um questionário que avalia os aspectos pedagógicos e de usabilidade de um jogo educacional. Alguns exemplos dos requisitos pedagógicos avaliados são: o conteúdo é coerente e contextualizado com a área e o nível de ensino propostos, esse conteúdo faz referência ao universo cotidiano dos alunos. Um exemplo de requisito de usabilidade citado é se o nível de concentração exigido está de acordo com o público do jogo.

O trabalho de Wu et al (2012) apresenta um estudo da arte sobre o uso de teorias da aprendizagem para os jogos educacionais. O trabalho configurou-se em uma Revisão Sistemática da Literatura (RSL) realizada em bases de dados (ACM Digital Library, ERIC, SAGE, ScienceDirect, SpringerLink, Taylor and Francis Online e Wiley). Dentre os estudos selecionados, 91 dos 658 estudos basearam a suas investigações em teoria da aprendizagem. O construtivismo (51\%) foi a teoria mais citada, seguida pelo humanismo, com $22 \%$, o cognitivismo, com $14 \%$, e por fim o behaviorismo, com apenas $13 \%$, sendo essa a teoria menos utilizada.

O trabalho de Ribeiro et al (2015) realizou um estudo semelhante ao de Wu et al (2012), porém os autores pesquisaram sobre teorias de aprendizagem aplicadas em jogos educacionais desenvolvidos no Brasil, com foco no ensino básico. O Google acadêmico foi utilizado e unitermos em português. A RSL selecionou 27 trabalhos, dentre eles $40,74 \%(n=11)$ não citam claramente qual a teoria de aprendizagem utilizada, a teoria de Piaget (construtivismo) está presente na maior parte dos trabalhos, com 22,22\% ( $n=6)$. Já a teoria de Vygotsky foi citada em 14,81\% $(n=4)$ dos trabalhos. Outros teóricos foram citados, como: Bloom $(7,40 \% ; n=2)$; Ausubel $(3,70 \% ; n=1)$; Papert $(3,70 \% ; n=1)$; Gardner $(3,70 \% ; n=1)$; Paulo Freire $(3,70 \% ; n=1)$; Teresa Colomer $(3,70 \% ; n=1)$; abordagem instrucionista, sem um teórico representante $(3,70 \%$; $\mathrm{n}=1)$. Vale salientar que houve combinações de teorias presentes em alguns trabalhos.

$\mathrm{O}$ estudo que se desenvolve no artigo em questão diferencia-se dos anteriores, pois ele não se limitou a pesquisar sobre jogos educacionais; a pesquisa aqui relatada identificou as teorias de aprendizagem utilizadas nos mais diversos tipos de SE, incluindo os Ambientes Virtuais de Aprendizagem (AVAs). Portanto, este trabalho unificou o termo para Softwares Educacional. A justificativa para incluir os AVAs na pesquisa é relatada pelo autor Brito (2003), ele afirma que:

É importante destacar que os ambientes virtuais de aprendizagem podem apresentar algumas ou todas as classificações de software educativo, de acordo com os tipos ou utilizações das ferramentas tecnológicas do mesmo. De maneira geral, os ambientes virtuais dispõem de ferramentas instrucionistas e construcionistas e são classificados de acordo com suas func ionalidades, basicamente, em softwares educativos do tipo perguntas e respostas, comunicação e cooperação (ex: Moodle).

Foram utilizados dois trabalhos como referência para realizar a RSL, a saber: Kitchenham e Charters (2007) e Lima (2012). O objetivo da pesquisa é identificar quais 
as teorias de aprendizagem (referenciadas às vezes como teorias cognitivas ou abordagens pedagógicas) são utilizadas pelos diversos tipos de SEs. Além disso, procurou-se investigar quais os requisitos dos SEs estão associados às teorias de aprendizagem (chamados de requisitos pedagógicos por este trabalho). Por fim, foram identificadas diversas formas de avaliação dos SE diante de vários aspectos, a saber: desempenho do aluno (aprendizagem), usabilidade do software, dentre outros.

O resto deste artigo está organizado da seguinte forma. A Seção 2 descreve a metodologia utilizada e as etapas da RSL. A Seção 3 descreve os resultados obtidos após a RSL. A Seção 4 detalha as considerações finais e trabalhos futuros.

\title{
2. Metodologia
}

Esta pesquisa tem caráter exploratório, configurando-se em uma Revisão Sistemática na Literatura. O trabalho de Kitchenham e Charters (2007) afirma que:

\begin{abstract}
Uma revisão sistemática da literatura é uma forma de avaliar e interpretar toda a pesquisa disponível relevante para a questão de pesquisa particular, área temática, ou fenômeno de interesse. As revisões sistemáticas têm como objetivo apresentar uma avaliação justa de um tema de pesquisa, utilizando uma metodologia confiável.
\end{abstract}

Portanto, o protocolo utilizado nesta pesquisa foi elaborado com informações baseadas no trabalho de Kitchenham e Charters (2007). Ele está dividido em três fases, a saber: planejamento, condução e documentação. Na fase de planejamento foram definidas: questões de pesquisa, fontes de busca e critérios de inclusão e exclusão. Na fase de condução foi realizada a seleção dos trabalhos; para isso utilizaram-se as estratégias de busca. Logo após, foi realizada a extração dos dados, que ocorreu durante a leitura dos artigos selecionados. $\mathrm{Na}$ fase de documentação os dados foram sintetizados, e o relatório foi gerado. As subseções a seguir irão descrever de forma detalhada cada fase.

\subsection{Planejamento}

\section{- Questões de pesquisa}

Para alcançar os objetivos desta pesquisa foram elaboradas quatro questões de pesquisa (QP), são elas:

QP1. Quais são os requisitos presentes nos mais diversos tipos de Softwares Educacionais, e quais deles são associados às teorias de aprendizagem?

QP2. Quais são as teorias de aprendizagem incluídas no processo de desenvolvimento de Softwares Educacionais?

QP3. Quais são as teorias de aprendizagem incluídas no processo de avaliação de Softwares Educacionais?

QP4. Quais as diversas formas de avaliar os Softwares Educacionais?

\section{- $\quad$ Fonte de busca}

Para esta RSL levou-se em consideração a busca manual e automática. Os engenhos de busca utilizados na busca automática foram: ACM Digital Library, IEEEXplorer e Science Direct. Na busca manual, os anais foram selecionados em eventos nacionais como: Simpósio Brasileiro de Informática na Educação (SBIE), o Workshop sobre Educação em Computação (WEI), o Worskhop de Informática na Educação (WIE), e periódicos da Revista Brasileira de Informática na Educação (RBIE) e Revista Novas Tecnologias na Educação (RENOTE). 
A busca manual é importante porque ela pode diminuir problemas de indexação dos artigos que estão localizados nas bibliotecas digitais, e podem existir outros estudos relevantes que não utilizam as palavras chaves que foram previstas nesta RSL.

\section{- Critérios de inclusão e exclusão}

A inclusão dos trabalhos é determinada pela relevância em relação às questões levantadas. Os critérios de exclusão foram definidos para deixar claro quais trabalhos são irrelevantes a essa pesquisa, eles estão detalhados na Tabela 1.

Tabela 1 - Critérios de Inclusão e exclusão

\begin{tabular}{|l|l|}
\hline \multicolumn{1}{|c|}{ Critérios de Inclusão (CI) } & \multicolumn{1}{c|}{ Critérios de exclusão (CE) } \\
\hline $\begin{array}{l}\text { CI1 - Publicações que evidenciam os requisitos e/ou } \\
\text { teorias de aprendizagem e/ou formas de avaliação de } \\
\text { qualquer tipo de SE (incluindo os AVAs). }\end{array}$ & $\begin{array}{l}\text { CE1 - Trabalhos que estão disponíveis na forma de } \\
\text { resumos, apresentações, entrevistas e estudos } \\
\text { secundários (Ex: revisões sistemáticas da literatura e } \\
\text { mapeamentos sistemáticos). }\end{array}$ \\
\hline $\begin{array}{l}\text { CI2 - Trabalhos que estão disponíveis para downloads } \\
\text { na rede da Universidade UFPE - CIn }\end{array}$ & $\begin{array}{l}\text { CE2 Artigos que não apresentam a especificação das } \\
\text { características do SE (incluindo os AVAs). }\end{array}$ \\
\hline CI3 - Publicações entre 2010 á 2015 & $\begin{array}{l}\text { CE3 - Publicações que evidenciem os requisitos e/ou } \\
\text { teorias de aprendizagem e/ou formas de avaliação dos } \\
\text { Objetos de Aprendizagem (OA), não serão consideradas. }\end{array}$ \\
\hline CI4 - Artigos em inglês, português ou espanhol. & $\begin{array}{l}\text { CE4 - Publicações que tratem dos SEs voltados para } \\
\text { áreas bem específicas, como: TV Digital e Realidade } \\
\text { Aumentada. }\end{array}$ \\
\hline & $\begin{array}{l}\text { CE5 - Trabalhos duplicados serão excluídos (o mais } \\
\text { completo será considerado) }\end{array}$ \\
\hline
\end{tabular}

\subsection{Condução}

\section{- $\quad$ Estratégia de busca e seleção dos trabalhos}

Para a busca automática foi elaborada uma string de busca; ela foi baseada em termos importantes com variações significantes, fundamentados nas perguntas de pesquisas. $\mathrm{O}$ conector $\mathrm{OR}$ foi utilizado para combinar os termos alternativos e o conector AND para ligar as palavras chaves. Portanto, a string de busca utilizada foi: ("software development" OR "software engineering" OR "software evaluation" OR "requirements") AND ("educational software" OR "educational games" OR "ubiquitous learning" OR "mobile learning" OR "blended learning" OR "e-learning" OR "intelligent learning environment" OR "Learning Environment").

A seleção dos trabalhos foi conduzida por buscas manuais e automáticas. Ambas as estratégias seguiram três etapas, a saber: (i) leitura do título, resumo e palavras-chave (se houver); (i) leitura da introdução e conclusão e exclusão dos trabalhos duplicados; (iii) leitura completa dos trabalhos e extração dos dados.

Inicialmente foram retornados (engenhos de busca) e estavam disponíveis (eventos nacionais e periódicos) 1715 artigos, após passar pelas três etapas da RSL foram selecionados 172 estudos primários. A lista completa de artigos analisados está disponível em: https://goo.gl/YdZbX7

\section{- Extração dos dados}

Os dados foram extraídos e organizados em uma planilha, contendo os seguintes campos: identificador ( $\mathrm{EP}=$ Estudo Primário), ano de publicação, fonte da publicação, local da publicação (considerando o primeiro autor), referência do trabalho e respostas das questões de pesquisa.

A fase de documentação será descrita na Seção 3, tendo em vista que ela faz parte dos resultados gerados após a síntese dos dados. 


\subsection{Ameaças à validade}

O protocolo da RSL seguiu etapas bem definidas, porém seguem algumas limitações encontradas:

- Se a pesquisa aqui proposta for realizada em datas ou usuários distintos, os resultados podem ser outros;

- Caso outras bases de dados venham ser utilizadas, os trabalhos retornados podem ser distintos ao apresentado nesta pesquisa;

- A string de busca utilizada pode não contemplar todos os sinônimos existentes para os termos identificados, sendo insuficiente para capturar todos os trabalhos relevantes;

Vale salientar que esta pesquisa utilizou a rede interna da UFPE- CIn para capturar os trabalhos disponíveis nas bases de dados.

\section{Resultados e discussão}

\subsection{Síntese dos dados}

Com o objetivo de organizar e facilitar a síntese dos dados, cada estudo primário foi organizado de acordo com a fonte de publicação do ano mais atual (2015) para o mais antigo (2010). Logo após, as informações foram sendo preenchidas e deram origem aos requisitos, teorias de aprendizagem utilizadas no desenvolvimento e avaliação dos SEs, e diversas maneiras de avaliar os SEs. Uma aplicação web ${ }^{1}$ foi utilizada para calcular a frequência em que os requisitos foram citados pelos artigos.

As Figuras 1 e 2 apresentam respectivamente a distribuição da amostra por ano de publicação e por fonte de busca. Observando a Figura 1, é possível perceber que o maior número de publicações (44 artigos) foi em 2014. Vale enfatizar que em 2015 houve apenas dois artigos (disponíveis na ACM e Science Direct), pois a RSL foi realizada entre abril e julho de 2015, e nenhum dos eventos e periódicos tinham disponibilizado a edição de 2015. Em relação à fonte de busca (Figura 2), o maior número de publicações foi na RENOTE, com 55 artigos incluídos na RSL.

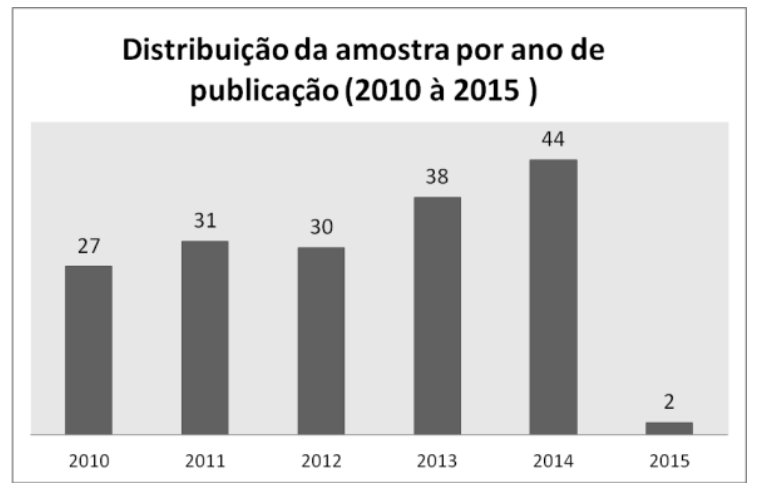

Figura 1- Distribuição dos artigos por ano ano de publicação

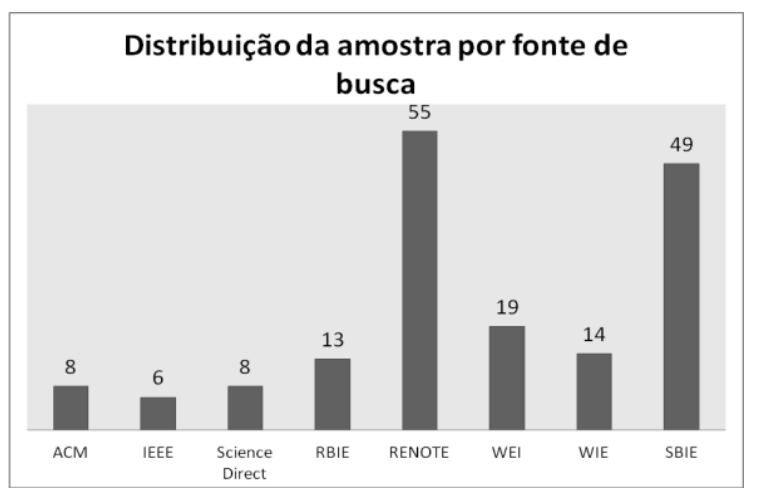

Figura 2- Distribuição dos artigos por fonte de busca

\subsection{Respostas às questões de pesquisa}

A primeira questão de pesquisa (QP1) faz o seguinte questionamento: Quais são os requisitos presentes nos mais diversos tipos de Softwares Educacionais, e quais deles são associados às teorias de aprendizagem?

\footnotetext{
${ }^{1}$ http://tagfest.com.br/wordranking/
} 
A Tabela 2 apresenta os dez requisitos mais citados pelos estudos primários. Percebe-se que o feedback (53 citações) é o requisito mais utilizado nos SEs, seguido pela pontuação (32 citações). Os requisitos mais citados são utilizados em jogos educacionais, pois $41 \%$ dos artigos analisados são deste domínio.

Tabela 2 - Dez requisitos mais utilizados no desenvolvimento de SE

\begin{tabular}{|l|l|c|}
\hline Requisito & \multicolumn{1}{|c|}{ Variação (ões) de termo(s) } & N* $^{*}$ \\
\hline Feedback & Feedback em libras; Feedback visual; Feedback personalizado. & 53 \\
\hline Pontuação & Pontos & 32 \\
\hline Níveis & $\begin{array}{l}\text { Níveis de conteúdos; Níveis de dificuldade; Níveis de interação; Níveis (Hierárquico); } \\
\text { Níveis de habilidade; }\end{array}$ & 31 \\
\hline Tempo & Tempo de digitação; & 22 \\
\hline Textos & Textos explicativos; Textos (representando a fala do avatar); & 19 \\
\hline Dicas & Dicas em libras; Dica sonora. & 18 \\
\hline & $\begin{array}{l}\text { Dúvidas; Assistente de dúvidas; Teleporte (Contato); Sistema sugere ajuda; Suporte } \\
\text { técnico; Atender aos visitantes; Fale conosco; Formulário para o usuário expressar } \\
\text { dúvidas; Formulário para o usuário propor soluções; helpfulness; Notificar via SMS } \\
\text { (resposta da dúvida do aluno); Oferecer apoio no desenvolvimento de projetos. }\end{array}$ & 17 \\
\hline Ranking & Não há variação & 16 \\
\hline Desafio & $\begin{array}{l}\text { Desafios (Jogabilidade); Missão; Missões em andamento; Missões concluídas; Painel } \\
\text { de desafios; Resolver problemas; Responder desafio; Enigma. }\end{array}$ & 15 \\
\hline Imagens & Imagens coloridas. & 14 \\
\hline N* Quantidade de vezes que o requisito foi citado pelos estudos primários & 17 \\
\hline
\end{tabular}

A Tabela 3 exemplifica alguns requisitos pedagógicos que foram identificados. Foi possível associar o requisito com o conceito da teoria de aprendizagem proposta. Observando a tabela, percebe-se que alguns termos só foram utilizados nos trabalhos sobre AVAs; eles estão em negrito e identificados com o símbolo (A). Essa é uma evidência que os SEs e AVAs possuem características semelhantes e em alguns casos compartilham o mesmo requisito.

Tabela 3 - Exemplo de alguns requisitos pedagógicos

\begin{tabular}{|c|c|c|}
\hline Requisito pedagógico & Variação (ões) de termo(s) & $\begin{array}{c}\text { Teoria ou método de } \\
\text { aprendizagem }\end{array}$ \\
\hline $\begin{array}{l}\text { ASG01 - Organizar o } \\
\text { conteúdo de forma } \\
\text { hierárquica }\end{array}$ & $\begin{array}{l}\text { Variações de termos: Dividido em módulos; } \\
\text { Representar o conteúdo em } \quad \text { árvore } \\
\text { hierárquica; Conteúdo em módulos; Conteúdo padrão } \\
\text { dividido por módulo; Organizar materiais; } \\
\text { (A) Exercícios associados ao módulo; Módulos; } \\
\text { Níveis (Hierárquico) }\end{array}$ & $\begin{array}{c}\text { Aprendizagem } \\
\text { Significativa (ASG) }\end{array}$ \\
\hline $\begin{array}{l}\text { ABP01 - Apoiar o } \\
\text { trabalho colaborativo }\end{array}$ & $\begin{array}{l}\text { Termo utilizado: (A) Oferecer apoio ao trabalho } \\
\text { colaborativo de desenvolvimento de trabalhos } \\
\text { (projetos). }\end{array}$ & $\begin{array}{l}\text { Aprendizagem Baseada } \\
\text { em Projetos (ABP) }\end{array}$ \\
\hline $\begin{array}{l}\text { PBL01 - Dividir grupo } \\
\text { por afinidades }\end{array}$ & Não há variação & $\begin{array}{c}\text { Aprendizagem Baseada } \\
\text { em problemas (do inglês, } \\
\text { Problem-Based Learning } \\
\text { (PBL) }\end{array}$ \\
\hline $\begin{array}{l}\text { CONS01 - Mostrar } \\
\text { feedback construtivo }\end{array}$ & $\begin{array}{l}\text { Variações de termos: Feedback; Feedback visual; } \\
\text { Mensagens; Mensagem com dicas; Mensagens } \\
\text { personalizadas }\end{array}$ & Construtivismo (CONS) \\
\hline $\begin{array}{l}\text { COMP17 - Ordem } \\
\text { cronológica }\end{array}$ & $\begin{array}{l}\text { Variações de termos: Organizar aulas } \text { (ordem } \\
\text { cronológica); Linearity; Disponibilizar aulas (ordem } \\
\text { cronológica) }\end{array}$ & $\begin{array}{l}\text { Comportamentalismo } \\
\text { (COMP) }\end{array}$ \\
\hline
\end{tabular}




\begin{tabular}{|c|c|c|}
\hline $\begin{array}{l}\text { SOC01 - Disponibilizar } \\
\text { formas de comunicação }\end{array}$ & $\begin{array}{l}\text { Variações de termos: Enquete ao vivo; Chat; } \\
\text { Ferramentas síncronas e assíncronas; Comunicação } \\
\text { através de Bluetooth; Comunicação por voz; } \\
\text { Comunicação } \quad \text { wireless; Comunicações; (A) } \\
\text { Comunidade; } \quad \text { Bate-Papo; Disponibilizar } \\
\text { ferramentas de Comunicação (entre alunos e } \\
\text { tutores); Email; Ferramenta conversação em tempo } \\
\text { real; Ferramentas interativas; Fórum; Mensagens; } \\
\text { Realizar seminários na web; Receber email; Sala de } \\
\text { bate-papo; Sala virtual; Troca de mensagens } \\
\text { (Alunos e professores) }\end{array}$ & $\begin{array}{l}\text { Sócio-Interacionismo } \\
\text { (SOC) }\end{array}$ \\
\hline $\begin{array}{l}\text { TC01- Objetos do } \\
\text { cotidiano }\end{array}$ & $\begin{array}{l}\text { Variações de termos: Objetos do cotidiano (Ex: pedras; } \\
\text { lago; chave); Imagens do cotidiano }\end{array}$ & $\begin{array}{l}\text { Teoria do Conhecimento - } \\
\text { Paulo Freire (TC) }\end{array}$ \\
\hline $\begin{array}{l}\text { TX01- Propor diversos } \\
\text { níveis de conteúdos }\end{array}$ & Não há variação & $\begin{array}{l}\text { Taxonomia de Bloom } \\
\text { (TX) }\end{array}$ \\
\hline $\begin{array}{l}\text { TAM01 - Recursos } \\
\text { multimídia }\end{array}$ & $\begin{array}{l}\text { Variações de termos: Animação; Tecnoteca; Vídeo } \\
\text { aulas; Videoteca; Webquests; Wikis Podcasts; } \\
\text { Simulações; Vídeos tutoriais; } \\
\text { (A) Gráficos animados; Media Channels Variety; } \\
\text { Apresentações narradas; Vídeo institucional; Vídeos } \\
\text { e imagens (Redes sociais); Videoteca; Visualizar } \\
\text { vídeo em tempo real; Biblioteca Digital. }\end{array}$ & $\begin{array}{l}\text { Teoria da Aprendizagem } \\
\text { Multimídia (TAM) }\end{array}$ \\
\hline $\begin{array}{l}\text { TIMU01- Prover } \\
\text { adaptação }\end{array}$ & $\begin{array}{l}\text { Variações de termos: Diferentes dinâmicas de } \\
\text { aprendizagem; Modelo híbrido; Interface adaptável; } \\
\text { Ambiente de Aprendizagem adaptativo; Atividades } \\
\text { (Alternativas: falsa e verdadeira); Atividades discursiva; } \\
\text { Atividades de múltipla escolha (A) Personalizar os } \\
\text { cursos existentes; Recomendação material; } \\
\text { Recomendações; Recomendar Conteúdos; } \\
\text { Conteúdos personalizados; Diferentes formas de } \\
\text { visualizar os resultados; Fornecer recomendações } \\
\text { por mensagem de texto. }\end{array}$ & $\begin{array}{l}\text { Teoria das Inteligências } \\
\text { Múltiplas (TIM) }\end{array}$ \\
\hline
\end{tabular}

A pergunta de pesquisa dois (QP2) faz o seguinte questionamento: quais são as teorias de aprendizagem incluídas no processo de desenvolvimento dos Softwares Educacionais? Os dados foram analisados separadamente (SEs e AVAs), mesmo com a generalização do termo (Softwares Educacionais). Dentre os 172 estudos primários selecionados, apenas 48 () citaram claramente a teoria de aprendizagem utilizada. Vale enfatizar que alguns trabalhos citam mais de uma teoria. A Figura 3 apresenta as teorias de aprendizagem usadas no desenvolvimento dos SEs; a teoria mais citada é o construtivismo (13 citações). Já a Figura 4 mostra as teorias mais usadas no desenvolvimento dos AVAs, são elas: construtivismo e sócio-interacionismo (5 citações). Portanto, o construtivismo é a mais utilizada (18 citações no geral). Vale enfatizar que este trabalho está generalizando o termo teoria de aprendizagem, pois Aprendizagem Baseada em Projetos, Aprendizagem Baseada em Problemas e Projetos (ABPP) e Aprendizagem baseada em Problemas, são considerados métodos de ensino (aprendizagem). 


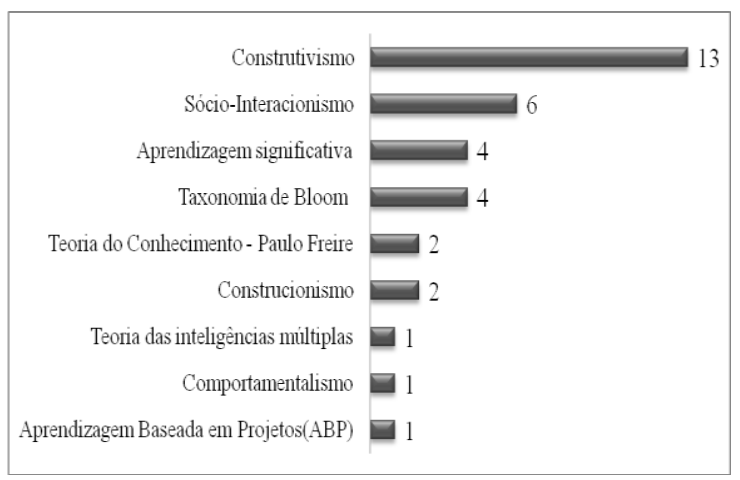

Figura 3 - Teorias de aprendizagem usadas no desenvolvimento dos SEs

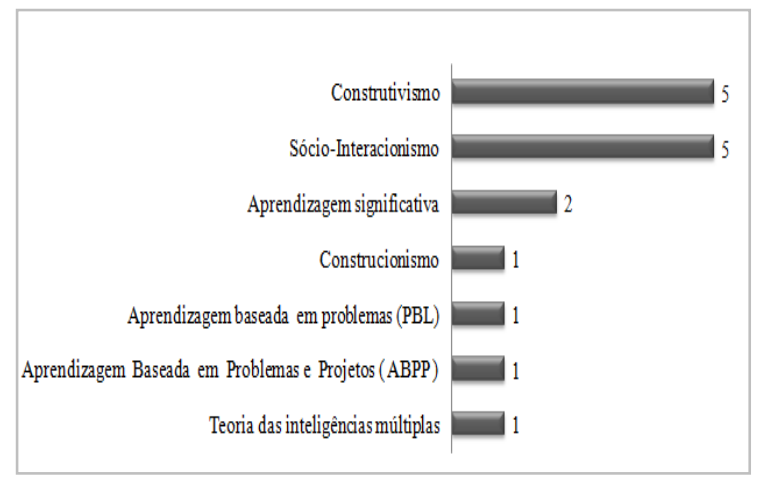

Figura 4 - Teorias de aprendizagem usadas no desenvolvimento dos AVAs

A pergunta de pesquisa três (QP3) faz o seguinte questionamento: quais são as teorias de aprendizagem incluídas no processo de avaliação dos Softwares Educacionais? A Figura 5 apresenta as teorias de aprendizagem utilizadas na avaliação dos SEs, a taxonomia de Bloom (3 citações) é a mais utilizada. A Figura 6 apresenta as teorias usadas na avaliação dos AVAs; vale enfatizar que os trabalhos sobre LMS (do inglês Learning Management System) e software E-learning foram incluídos nesta categoria; a teoria mais usada na avaliação dos AVAs é o sócio-interacionismo (4 citações).

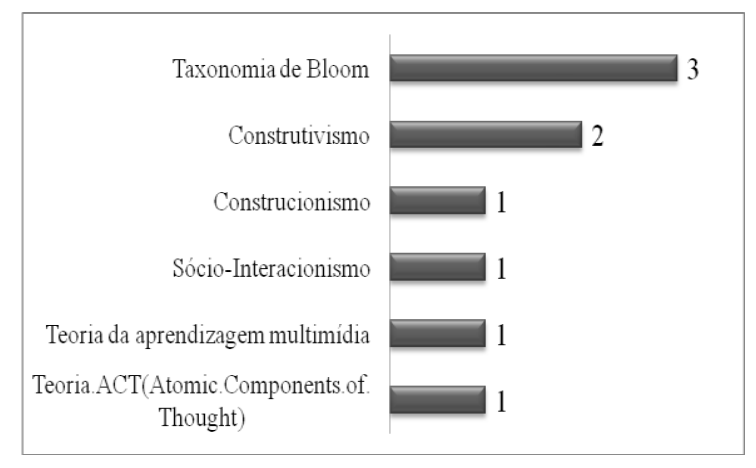

Figura 5 - Teorias de aprendizagem usadas na avaliação dos SEs

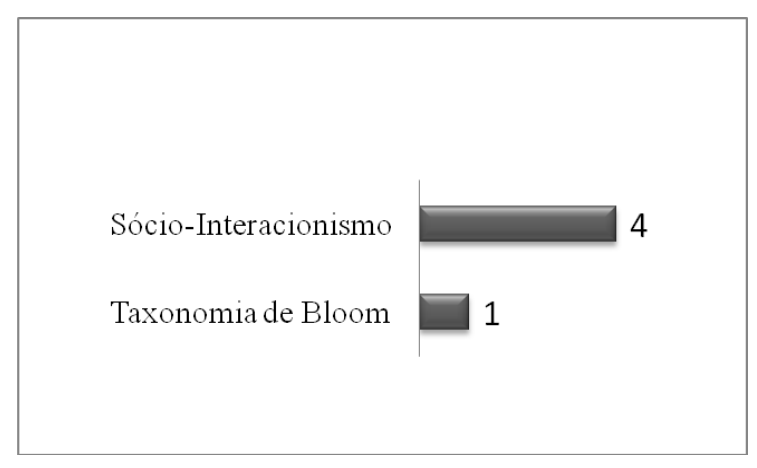

Figura 6 - Teorias de aprendizagem usadas na avaliação dos AVAs

A quarta pergunta de pesquisa (QP4) faz o seguinte questionamento: Quais as diversas formas de avaliar os Softwares Educacionais? A Tabela 4 mostra as diversas formas de avaliação em SEs; os dados estão dispostos separadamente (SEs e AVAs). Observando a Tabela 4, percebe-se que 35 artigos não realizaram avaliação no contexto do SEs, e a forma mais comum de avaliar SE é através de questionário (72 citações). Em relação aos AVAs, 18 artigos não realizaram avaliação, e o questionário (24 citações) também é a forma mais utilizada no processo de avaliação. Vale enfatizar que alguns trabalhos realizaram diversos tipos de avaliação nos seguintes aspectos: aprendizagem, usabilidade, acessos ao sistema, métodos empíricos, dentre outros.

Tabela 4 - Diversas formas de avaliar os SEs

\begin{tabular}{|c|c|c|c|}
\hline & \multicolumn{3}{|c|}{ Tipos de avaliação } \\
\hline & & Metodologia RETAIN & Método de Inspeção \\
& Não houve avaliação & (Relevância; Incorporação; & Semiótica (MIS) \\
& (1 citação) \\
Softwares & Transferência; Adaptação; & \\
\hline
\end{tabular}




\begin{tabular}{|c|c|c|c|}
\hline \multirow[t]{5}{*}{ Educacionais } & $\begin{array}{l}\text { Questionários } \\
\text { (72 citações) }\end{array}$ & $\begin{array}{c}\text { Testes de usabilidade (Técnica } \\
\text { Think Aloud) } \\
\text { ( } 2 \text { citações) }\end{array}$ & $\begin{array}{c}\text { Método de avaliação da } \\
\text { comunicabilidade } \\
\text { (MAC) } \\
\text { (1 citação) }\end{array}$ \\
\hline & $\begin{array}{l}\text { Experimento } \\
\text { (6 citações) }\end{array}$ & $\begin{array}{l}\text { Avaliar ações do usuário } \\
\text { (acertos, erros e tempo) } \\
\text { ( } 2 \text { citações); }\end{array}$ & $\begin{array}{l}\text { Avaliação quantitativa } \\
\text { (através do uso do } \\
\text { google Analytics- } \\
\text { QtdeAcesso) } \\
\text { (1 citação) }\end{array}$ \\
\hline & $\begin{array}{l}\text { Observação } \\
\text { (3 citações) }\end{array}$ & $\begin{array}{c}\text { MetodologiaLORI (Learning } \\
\text { Object ReviewInstrument) e } \\
\text { metodologia GameFlow } \\
\text { (1 citação) }\end{array}$ & $\begin{array}{c}\text { Medir emoções (método } \\
\text { Emocard) } \\
\text { (1 citação) }\end{array}$ \\
\hline & $\begin{array}{l}\text { Entrevista com os usuários } \\
\text { (1 citação) }\end{array}$ & $\begin{array}{c}\text { Simulação para comparar } \\
\text { parâmetros já divulgados na } \\
\text { literatura. } \\
\text { (1 citação) }\end{array}$ & $\begin{array}{l}\text { Prototipagem } \\
\text { (1 citação) }\end{array}$ \\
\hline & $\begin{array}{l}\text { Dez critérios técnicos e } \\
\text { pedagógicos } \\
\text { (1 citação) }\end{array}$ & $\begin{array}{l}\text { Framewor }(\text { EFEX) } \\
\text { (1 citação) }\end{array}$ & \\
\hline \multirow{5}{*}{$\begin{array}{c}\text { Ambientes } \\
\text { Virtuais de } \\
\text { Aprendizagem }\end{array}$} & $\begin{array}{l}\text { Não houve avaliação } \\
\text { (18 artigos) }\end{array}$ & $\begin{array}{l}\text { Modelos markovianos } \\
\text { ( } 2 \text { citações) }\end{array}$ & $\begin{array}{c}\text { Inspeção baseada em } \\
\text { taxonomia (Log dos } \\
\text { usuaríos) (1 citação) }\end{array}$ \\
\hline & $\begin{array}{l}\text { Questionário } \\
\text { (24 citações) }\end{array}$ & $\begin{array}{c}\text { Avaliação da aprendizagem } \\
\text { através da criação de mapas } \\
\text { conceituais } \\
\text { (1 citação) }\end{array}$ & $\begin{array}{c}\text { Métricas para avaliação } \\
\text { (NBR (ISO/IEC 14598); } \\
\text { NBR (ISO/IEC 9126-1) } \\
\text { (1 citação) }\end{array}$ \\
\hline & $\begin{array}{l}\text { Modelo de } 3 \text { parâmetros da } \\
\text { teoria da resposta ao Item } \\
\text { (avalia o desempenho do } \\
\text { aluno) (1 citação) }\end{array}$ & $\begin{array}{l}\text { Modelo (ASIBPq) } \\
\text { (1 citação) }\end{array}$ & $\begin{array}{l}\text { Observação } \\
\text { (1 citação) }\end{array}$ \\
\hline & $\begin{array}{c}\text { Avaliação sócio interacionista } \\
\text { (1 citação) }\end{array}$ & $\begin{array}{l}\text { Grupo focal } \\
\text { ( } 2 \text { citações) }\end{array}$ & $\begin{array}{c}\text { Software estatístico } \\
\text { SPSS e Teste T } \\
\text { (1 citação) } \\
\end{array}$ \\
\hline & $\begin{array}{l}\text { Experimento } \\
\text { ( } 2 \text { citações) }\end{array}$ & $\begin{array}{c}\text { Gravar as ações do usuário } \\
\text { durante a interação com o } \\
\text { sistema. } \\
\text { (1 citação) }\end{array}$ & $\begin{array}{l}\text { Estudo de caso } \\
\text { (1 citação) }\end{array}$ \\
\hline
\end{tabular}

\section{Considerações finais}

Este artigo apresentou uma Revisão Sistemática da Literatura. O seu objetivo principal foi identificar as teorias de aprendizagem usadas pelos SEs; vale enfatizar que os trabalhos sobre AVAs foram incluídos na pesquisa, tendo em vista que eles contêm várias características semelhantes aos SEs (Brito 2013). A amostra analisada nesta pesquisa (172 artigos) constatou que apenas 48 trabalhos $(27,9 \%)$ relatam o uso de alguma teoria da aprendizagem.

Os resultados também comprovaram que a teoria de aprendizagem mais usada pelos SEs é o construtivismo de Piaget, corroborando com os trabalhos de Wu (2012) e Ribeiro (2015), pela preferência da teoria de aprendizagem construtivista. Em contrapartida, no contexto dos AVAs, o sócio-interacionismo de Vygostky é a teoria mais citada.

Os resultados mostraram que 2014 foi o ano com mais publicações relevantes (44 artigos) a essa pesquisa, e a RENOTE é a fonte de busca com mais artigos incluídos (55 artigos) na RSL. Além disso, foi possível identificar os principais requisitos para SEs, no qual o feedback (53 citações) é bastante utilizado. Além disso, foi possível identificar dez requisitos pedagógicos associados a dez tipos de teorias/métodos de aprendizagem (Ver tabela 3). Em relação à avaliação dos SE, o questionário é o mais utilizado, talvez pela facilidade de aplicação e por ter um baixo custo. 
Alguns trabalhos da literatura criticam a baixa qualidade dos SE, e evidenciam a dificuldade que o professor tem de utilizá-lo nas aulas (Hinostroza, 2001). "Um dos motivos dessa ineficácia pode ser o fato do design de suas interfaces considerar poucos aspectos do processo de aprendizagem, privilegiando aspectos estruturais das interfaces."(Gomes, 2008). Portanto, é preciso repensar o processo de desenvolvimento dos SEs, para proporcionar um equilíbrio entre os requisitos técnicos e pedagógicos.

Esta pesquisa está em andamento, os requisitos identificados foram organizados em forma de catálogo, inicialmente foram identificados 112 requisitos, dentre eles, 67 são requisitos pedagógicos (baseados em teorias e/ou métodos de aprendizagem), 15 são requisitos de usabilidade e 30 são requisitos gerais. Um survey foi realizado para validar a versão inicial do catálogo, 29 especialistas em informática na educação participaram nessa primeira etapa, os dados gerados tem natureza quantitativa e qualitativa. Com os resultados foi possível validar a relevância dos requisitos existentes no catálogo e identificar outros requisitos que não estavam presentes. Um dos trabalhos futuros é gerar uma nova versão do catálogo, visando a qualidade do mesmo no desenvolvimento dos SEs.

\section{Referências bibliográficas}

AN. D.Y; SILVA. C. D; RIBEIRO. D. M.G; ROCHA. P. B. R.; MALTINTI. C.; NUNES. V. B.; FÁVERO, R.. Digita - um Jogo Educativo de Apoio ao Processo de Alfabetização Infantil. In: Anais do Simpósio Brasileiro de Informática na Educação, Vol. 24. No 1. 2013

BRITO. L.M; GIUBERTI. J. R. J; GOMES. S. G. S; MOTA. J. B. Ambientes virtuais de aprendizagem como ferramentas de apoio em cursos presenciais e a distância. RENOTERevista Novas Tecnologias na Educação V. 11 N$^{\circ}$ 1, julho. 2013

FERREIRA. B.M; RIVERO. L; LOPES. A; MARQUES. A.B; CONTE.T. UsabiliCity: Um Jogo de Apoio ao Ensino de Propriedades de Usabilidade de Software Através de Analogias. In: Anais do Simpósio Brasileiro de Informática na Educação, Vol. 25, $\mathrm{N}^{\mathrm{o}}$ 1, p. 1273-1282. 2014

GOMES. A. S. Referencial Teórico Construtivista para Avaliação de Software Educativo.

Revista Brasileira de Informática na Educação. V. 16 N$^{\circ} 2.2008$

GOMES, A. S., PADOVANI, S. Usabilidade no ciclo de desenvolvimento de software educativo. In: Anais do Simpósio Brasileiro de Informática na Educação, 2005

HINOSTROZA J. E.; MELLAR.H. Pedagogy embedded in educational software design: report of a case study, Comp. \& Education, v. 37, n. 1, p. 27-40, 2001

KITCHENHAM, B., CHARTERS S. Guidelines for performing Systematic Literature Reviews in Software Engineering. Technical Report, EBSE-2007-01, School of Computer Science and Mathematics, Keele University, 2007

LIMA. F. A. Métodos, Técnicas e Ferramentas para o Desenvolvimento de Software Educacional: Um Mapeamento Sistemático, no estado de Pernambuco. Recife CIn/UFPE, 2012. Dissertação de mestrado.

RIBEIRO. R. J.; SILVA .N. J.; FRASSON.A.C.; PILATTI.L.A.; SILVA. S. C. R. Teorias de Aprendizagem em Jogos Digitais Educacionais: um Panorama Brasileiro. RENOTE Revista Novas Tecnologias na Educação, V. 13 Nº 1, julho, 2015.

TCHOUNIKINE, P. Computer Science and Educational Software Design. A Resource for Multidisciplinary Work in Technology Enhanced Learning 1st Edition., 2011, XIII, 180 p. 1 illus.

WU. WH.; Chiou. WB.; Kao. HY.; Hu. CHA.; Huang. SH. Re-exploring game-assisted learning research: The perspective of learning theoretical bases. Computers \& Education, v. 59, n. 4, p. 1153-1161. 2012. 